\section{Factors Contributing to Non-Compliance with Feed- ing Practices by HIV Positive Women in Limpopo Province, South Africa}

\author{
Patrone Rebecca Risenga* \\ Department of Health Studies, University of South Africa, Gauteng Prov- \\ ince, South Africa
}

\begin{abstract}
South Africa consists of many cultural groups who manage pregnancy and lactation differently. Infant nutrition is one of the most important practices to promote life and reduce the escalating infant mortality rate. Globally, around 1.5 million women living with HIV become pregnant each year. Without antiretroviral drugs, up to $40 \%$ of their babies will also be infected with HIV. Regardless of all the efforts of giving Antiretroviral Therapy (ART) to HIV positive pregnant women, babies are still infected with HIV; children aged 0-4 years living with HIV face the highest risk of AIDS-related death compared with all other age groups because HIV progresses quickly in babies. The purpose of the study was to explore the factors contributing to non-compliance with feeding practices by HIV positive women in South Africa. A non-probability purposive sampling was used. The target population was females who are HIV positive nursing mothers on ART. Thirty nursing mothers between 20 and 35 years took part in the study. A qualitative research method was used. In-depth individual interviews were used for data collection. Tesch's approach was used for data analysis. Two themes emerged from the study, namely:

- Community reactions regarding infant feeding

- Physical problems experienced by mothers who are breastfeeding as some of the influential factors regarding infant feeding practices
\end{abstract}

Conclusion: Promoting the use of correct infant feeding options by HIV positive mothers and other members in the communities will lessen infant morbidity.

Keywords: AIDS; Exposed; HIV; Infant feeding practices

*Corresponding author: Patrone Rebecca Risenga, Department of Health Studies, University of South Africa, P.O. Box 392, Unisa 0003, Gauteng Province, South Africa, Tel: +27 0124296769; Fax: +27 0124296688; E-mail: risenpr@unisa.ac.za

Citation: Risenga PR (2017) Factors Contributing to Non-Compliance with Feeding Practices by HIV Positive Women in Limpopo Province, South Africa. J AIDS Clin Res Sex Transm Dis 4: 010.

Received: February 27, 2017; Accepted: May 17, 2017; Published: May 31, 2017

\section{Introduction}

HIV testing is vitally important in order to access treatment and knowledge of one's positive status can lead to behaviours to protect other people from infection [1]. HIV disease continues to be a serious health issue in parts of the world. Worldwide, there were about 2.1 million new cases of HIV in 2015. About 36.7 million people are living with HIV around the world and as of June 2016, 17 million people living with HIV were receiving medicines to treat HIV, called Antiretroviral Therapy (ART). In 2015, there were an estimated 17.8 million women living with HIV (aged 15 years and older), constituting 51\% of all adults living with HIV. HIV/AIDS is the leading cause of death among women of a reproductive age (aged 15-44). In 2015, of the total estimated 1.9 million new HIV infections globally, $47 \%$ were among women (Centers for Disease Control, 2016). In 2015, almost 8 out of 10 pregnant women living with HIV received antiretroviral medicines to prevent the transmission of HIV to their children.

An estimated 1.1 million people died from AIDS-related illnesses in 2015. Sub-Saharan Africa bears the heaviest burden of HIV/AIDS worldwide, accounting to $65 \%$ of all new HIV infections. Other regions significantly affected by HIV/AIDS include Asia, The Pacific, Latin America, the Caribbean, Eastern Europe and Central Asia. The total number of people living with HIV in South Africa was estimated at approximately 7.03 million in 2016 , making up $12.7 \%$ of the total population. For adults aged $15-49$ years, an estimated $18.9 \%$ of the population is HIV positive (mid-year population estimates, 2016).

Globally, around 1.5 million women living with HIV become pregnant each year. Without antiretroviral drugs, up to $40 \%$ of their babies will also become infected with HIV. Without treatment, half of these children will die before their second birthday. In 2015, almost 8 out of 10 pregnant women living with HIV received antiretroviral medicines to prevent the transmission of HIV to their children. Regardless of all the efforts of giving ART to HIV positive pregnant women, babies are still infected with HIV and children aged 0-4 years living with HIV face the highest risk of AIDS-related death compared with all other age groups because HIV progresses quickly in babies. MotherTo-Child Transmission (MTCT) accounts for over 90\% of new HIV infections among children. According to PMTCT guidelines (2015) in South Africa, all pregnant women-whether their HIV results are positive or negative-should be considered as part of the PMTCT programme and should be offered information on the availability of PMTCT interventions during all healthcare consultations. They must also be counselled on safe infant feeding, be assisted in making appropriate feeding choices and be informed and counselled that exclusive breastfeeding for the first six months is the best option. They should also be informed and counselled that complementary foods should only be introduced from 6 months of age, with continued breastfeeding for at least 12 months.

In 2016, UNAIDS with PEPFAR launched Start free, Stay free, AIDS free-a framework calling for a worldwide sprint towards "super fast-track targets" to end AIDS among children, adolescents and young women by 2020 [2]. Breastfeeding is the best method of feeding babies, whether exposed to HIV or not; however, good feeding 
options are crucial in the prevention of HIV infection from mothers to babies. An earlier study carried out in South Africa by Coovadia and Kindra showed that after 18 months of follow-up, infants who were exclusively breastfed were significantly less likely to become infected with HIV [3]. Mixed feeding, in which infants are exposed to breast milk and to other liquids, as well as solid foods and formula feeds or cow's milk, is associated with a higher rate of HIV transmission, probably due to exposure to allergens that irritate the gut and lead to inflammation, thus increasing the risk of HIV infection from breast milk [4]. Mixed feeding is very common in African countries and solid food may be introduced as early as before three months. A study conducted in Ethiopia reflected that some of the factors leading to mixed feeding include insufficient breast milk, which makes mothers go for mixed feeding. Another factor cited included a lack of fostered counselling and information about the risks and benefits of breastfeeding, resulting in mothers deciding to mix feed the babies while knowing that mother to child transmission of HIV virus can occur during pregnancy, delivery and breast milk feeding. However, other HIV positive women may have made suboptimal infant feeding decisions based only on the knowledge that HIV can be transmitted by breastfeeding and not knowing about the other routes. This can be done in an effort to prevent HIV transmission to their infants and thus women may be endangering their infants' health unintentionally. This reflects on the need for further counselling and offering of clear information about postpartum HIV transmission to all women seeking antenatal care [5]. Breastfeeding frequently continues alongside solid foods for 18 months after birth. These practices expose infants to HIV infection, hence the study was crucial and aimed at understanding breastfeeding and infant feeding practices in Vhembe District, Limpopo, South Africa.

\section{Research Objectives}

The objective of this study was to explore and describe the factors contributing to non-compliance with feeding practices by HIV positive women in Limpopo.

\section{Materials and Methods}

\section{Study design}

The research design was qualitative, descriptive and explorative in nature. The researcher used this approach to explore the factors contributing to non-compliance with feeding practices by HIV positive women in Limpopo.

\section{Study setting}

Participants interviewed were 30 in number; all were Venda speaking people who were interviewed in Xitsonga. The study took place in the clinics from three different villages in Vhembe District, Limpopo. Vhembe District Municipality is a category c municipality located in the northern part of Limpopo. It shares borders with Zimbabwe and Botswana in the north-west and Mozambique in the south-east through the Kruger National Park. The Limpopo River valley forms the border between the district and its international neighbours. The district includes the former Transvaal and areas that were previously under Venda and Gazankulu Bantustan's administration. It comprises four local municipalities: Musina, Thulamela, Makhado and Collins Chabane; Mutale District has been dissolved to fall under a new municipality developed. The district municipal offices are located in the town of Thohoyandou. It covers a geographical area that is predominantly rural. It is a legendary cultural hub and a catalyst for agricultural and tourism development. The map below depicts municipalities in Vhembe Districts (Figure 1).

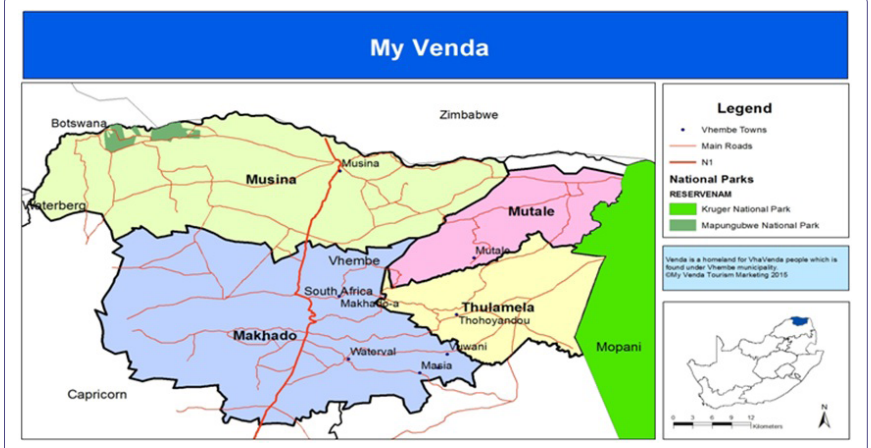

Figure 1: Vhembe District (Venda History).

Limpopo had a population estimate of 5,726,800 in 2016, which makes up $10.4 \%$ of the South African population.

\section{Population}

Population refers to all (entire set of individuals), or a complete set of persons that possess some common characteristics that are of interest to the researcher, or that people or a group of persons meet the criteria the researcher is interested in studying. The population for this study includes all HIV positive mothers on ART, is breastfeeding and resides in the Vhembe District in Limpopo.

\section{Target population}

The target population in this study included mothers who are HIV positive on ART and are breastfeeding.

\section{Sampling process}

All the participants were contacted according to the specified criteria for stakeholder analysis.

\section{Sample}

The study sampling method utilized was nonprobability sampling; elements were chosen from the population using purposive sampling. Purposive sampling was utilized for participants based on specified criteria.

\section{Sampling criteria}

The participants were selected based on the following criteria:

- Being HIV positive

- Breastfeeding mother with a baby between one month and one year

- On antiretrovirals for more than six months

- An adult of 18 years and above

\section{Size}

Participants interviewed were 30 females between 20 and 35 years who had new-born babies between one month and one year. All these mothers were on ART because in South Africa all pregnant women who are found to be HIV positive are given ART on diagnosis according to PMTCT guidelines and have received counselling. Sample size depended on data saturation and only 30 participants were interviewed-all HIV positive breastfeeding women on ART. After 30 interviews, the information kept being repeated, so it was no longer necessary to continue with the interviews. This is called data saturation. Rubin and Rubin (2011) referred to saturation as getting similar 
data or ideas over and over again during the course of interviewing in a qualitative study that signifies completion of data collection of a particular phenomenon.

\section{Data collection methods}

In this study, in-depth individual interviews were used in the form of unstructured interviews and field notes to collect data. The following question was asked of the participants: What are the factors contributing to non-compliance with feeding practices by HIV positive women in the Vhembe District of Limpopo Province, South Africa?

\section{Data collection process}

All 30 individual interviews were conducted at the clinic. The empty room at the clinic was used for data collection as a neutral venue. Research participants were eager to participate. Introduction of questions was done by the researcher and an audiotape was used with their permission. Each interview lasted between 45 and 60 minutes. All the participants were Tsonga speaking and were interviewed in Xitsonga. They were from three different villages in Vhembe District, Limpopo. Paraphrasing and summarizing of responses and probing for answers were used for data solicitation. During the interviews data saturation occurred after interviewing 30 women.

\section{Ethical considerations}

Ethical considerations were adhered to in order to protect the participants as presented in the subsequent descriptions. Ethical clearance was obtained from the University of Venda and the Tshilidzini Regional Hospital in the Vhembe District of Limpopo.

Participants also signed the consent form after a thorough explanation of the study. Possible voluntary withdrawal from the study at any point was emphasized. Confidentiality, anonymity and privacy were maintained through the use of codes instead of participants' names. Credibility was achieved through continual interaction and the researcher remained with the participants for three months during data collection. The researcher continuously played back the tapes to participants for them to validate what had been recorded. Two of the participants' transcripts were later read to confirm the truth. The researcher suspended information known to her with regard to infant feeding practices to avoid misinterpreting the phenomenon as being experienced by the participants. Bracketing made it possible for the researcher to focus on participants' views and to shape the data collection process according to it. The reason for bracketing was to reduce bias and the two aspects that led to the necessity of bracketing of any preconceived ideas the researcher could have were addressed.

\section{Data analysis}

In this study Tesch's eight steps as described by Creswell were used for data analysis [6]. Themes, categories and subcategories were developed according to the data obtained from individual interviews. The steps used during data analysis are as follows:

Step one: Getting a sense of the whole. This involved reading through all of the transcriptions carefully, jotting down ideas as they came to mind. The researcher went through all the transcriptions carefully in order to make sense of the transcriptions.

Step two: The researcher picked one document (interview) and went through it in order to check the meaning.

Step three: Topics were formed from the data obtained and clusters for similar topics were formulated.
Step four: The formulated tasks were abbreviated as codes and written next to the appropriate segments of the text. Verification was done to check whether new categories and codes had emerged.

Step five: Categories were formed from each theme that was developed and had been presented in the findings.

Step six: A final decision on the abbreviation for each category was made and codes were written alphabetically.

Step seven: Data material belonging to one category were assembled in one place and preliminary analysis was done.

Step eight: Existing data were recorded [6].

\section{Results and Discussion}

Two themes and six categories emerged from the data analysis as shown in table 1.

\begin{tabular}{|l|l|}
\hline Theme & Categories \\
\hline \multirow{4}{*}{$\begin{array}{l}\text { Community members' reactions } \\
\text { regarding infant feeding }\end{array}$} & $\begin{array}{l}\text { Failure to communicate HIV results to } \\
\text { loved ones coupled with denial of the } \\
\text { infection }\end{array}$ \\
\cline { 2 - 2 } & $\begin{array}{l}\text { Cultural beliefs regarding infant feeding } \\
\text { associated with HIV and AIDS }\end{array}$ \\
\cline { 2 - 2 } & Stigma and infant feeding practices \\
\hline \multirow{2}{*}{$\begin{array}{l}\text { Physical problems experienced by } \\
\text { mothers who are breastfeeding }\end{array}$} & $\begin{array}{l}\text { Sixed feeding reactions } \\
\text { breastfeed }\end{array}$ \\
\cline { 2 - 2 } & $\begin{array}{l}\text { Severe illness resulting in lack of } \\
\text { energy hampers feeding options for } \\
\text { the baby }\end{array}$ \\
\hline \multicolumn{2}{|c|}{ Table 1: Themes and categories. } \\
\hline
\end{tabular}

Theme 1: Community members' reactions regarding infant feeding fuelling the spread of HIV to infants and new-born babies as well as children

Discussions with participants revealed that mothers want to stick to feeding options that are healthy to avoid HIV infection of their babies. However, community members' reactions make it difficult for these mothers to follow safe infant feeding practices. During interviews, four categories emerged namely: Failure to communicate HIV results to loved ones coupled with denial of the infection, cultural beliefs make it difficult to adopt correct practices regarding infant feeding, stigma and infant feeding practices and mixed feeding reactions.

Failure to communicate HIV results to loved ones coupled with denial of the infection

Twenty-five out of thirty participants indicated that failure to disclose their positive HIV status made it difficult for them to use formula feeding or breastfeeding exclusively. The extracts below summarize the participants' views on lack of disclosure.

"Since I did not disclose my HIV positive status it will be suspicious if I start to use formula feeding" (participant 30-28 years).

"It was difficult for me not to breastfeed as I haven't disclosed, my mother in law questioned why I need formula feeding as I am staying at home and there are times when I am sick and I cannot breastfeed" (participant 1-20 years).

"I told my sister about my status from that day she turned her back from me so imagine what is going to happen when I tell my mother 
and father in law, when my own flesh and blood treat me like that" (participant 3-34 years).

Similar to Seidel, Sew Paul and Dano's study conducted in South Africa, which explored the experiences and decisions taken around breastfeeding by a peer support group of $13 \mathrm{HIV}$ positive mothers meeting at King Edward VIII Hospital, reasons for non-disclosure of HIV were related to confidentiality [7]. Laar, Ampofo, Tuakli and Quakyi's study indicated that one participant was highly stigmatized at home and in her area after disclosing her HIV status to her husband-a man she claimed is an alcohol addict [8]. Disclosure is not an easy thing to do, but is a process that requires adjustment and the ability to deal with the stigma on the side of a mother who is HIV positive. Other participants had difficulty accepting their HIV status-which was seen to have a detrimental effect on their life-not knowing whether to believe what was presented to them by health care workers regarding their HIV status as reflected underneath.

Participants in the study had difficulty understanding why they were infected with HIV and had some doubts regarding the condition. The participants' remarked:

"I find it difficult for me to understand how I got infected because the man I got married to was the first man to indulge in sexual intercourse with that's why I doubt the testing conducted at the clinic" (participant 13-29 years).

"Why me really is it punishment from God or what, I am failing to get to terms with it" (participant 25-28 years).

The findings of this study were supported by several studies regarding HIV denial as a problem in most people who are infected by HIV. Denial and lack of disclosure were perceived as barriers to using health institutions for HIV related problems, which creates more problems because clients will only visit the facility when they have health problems [9].

Piper, Enah and Daniel's study indicated that the majority of participants had average HIV knowledge levels and that HIV denial, stigma and misconceptions played a role in the current HIV/AIDS epidemic among rural African American adolescents [10]. Nurses and health care professionals can play a key role in understanding and addressing the HIV stigma, denial and misconceptions among people in South Africa to reduce HIV/AIDS statistics that are soaring in the country.

\section{Cultural beliefs regarding infant feeding associated with HIV and AIDS}

Culturally breastfeeding is an expectation. Formula feeding is shunned because it means the woman is not taking enough nutrients or the family is not feeding her properly. Some of the participants reported that they used the excuse that the hospital said they must both breastfeed and formula feed.

"Culturally when a baby cries it simply means he/she is hungry; a very fine and soft porridge containing herbal medicine is prepared and given to the baby. The herbal medicine in the soft porridge is for the fontanelle to close faster" (participant 19-35 years).

Another participant said: "I was breastfeeding my baby very well, however as early as before the end of the first month of my baby's life my mother indicated that I was must give the baby soft porridge because my family believed he is hungry hence he was crying most oftenly. I felt very sad because I knew that I am exposing my son to HIV" (participant 10-26 years).
"Culture forms the basis for our survival whatever they say is correct so who am I to deny the reasons for giving my daughter soft porridge when elders tell me to do, it does not matter what the nurses are saying about HIV because our ancestors will protect her from HIV evil spirits" (participant 15-30 years).

The finding of this study is that family and society play a crucial role and have influence on infant feeding methods. A study conducted in South Africa indicated that infant feeding is clearly a social practice, involving not only the directly participating mother and baby, but also the social context of that interaction [11]. This includes many other role players, for example, male partners and female relatives (particularly, older relatives such as mothers, mothers-in-law and aunts), who commonly exchange "lay knowledge" on the best way to feed babies [12]. Moreover, as highlighted by Zachariah et al., (2011) this context involves "gendered power relations within and beyond the household [that] are likely to have a key role in whether or not [and how] mothers engage with Prevention of Mother to Child Transmission (PMTCT) programmes", including infant feeding prescripts [11]. In addition, societal expectations and norms also exert a powerful influence. In Botswana, it was found that although formula feeding among HIV positive women was strongly encouraged by counsellors and formula was provided by the clinics, women went home only to practice mixed feeding [13]. This was attributed to influences from extended families and the community members. Similarly, Omari et al., reported in Zambia that HIV positive women changed to mixed feeding even though they started out with exclusive breastfeeding [14]. Once again, reasons included sociocultural and expectations of family members (partners and mothers-in-law, extended families) and community members [14]. In Tanzania, HIV positive mothers who were not able to do exclusively replacement feeding continued mixed feeding since they believed their milk was not enough to make the baby grow "fat and shiny" as expected by kin and neighbours [15]. A study that investigated infant feeding choices and the experiences of HIV positive mothers from two Ghanaian Districts, found social pressure and local norms were factors that influenced the mother's decisions to mix feed their children [8].

Studies have shown that exclusive breastfeeding, that is, giving the infant breast milk only and no other liquids or solids for the first six months of life, reduces the rate of Mother To Child Transmission (MTCT) to less than one quarter compared to mixed feeding and HIV-free survival of the infants $[3,16,17]$. It is therefore crucial that mothers be made aware of HIV transmission rates from mother to child with regard to mixed feeding if the world would want to reach an HIV negative generation by 2020 .

\section{Stigma and infant feeding practices}

Participants indicated that they experience stigma related to infant feeding practices from community members, which sometimes forces them to breastfeed babies while knowing that they are placing their babies at risk of contracting HIV.

One participant said: "I have seen the treatment given to a woman who has indicated that she is infected, each time she goes to community gatherings nobody wants to sit next to her, it's so pathetic, so I end up giving my baby bottle when I am alone and breastfeed when I am in the midst of other people" (participant 2-23 years).

Another quote from a participant: "I am forced to breastfeed my child for long time regardless of my HIV status because everybody knows nowadays that once a woman delivers and does not breastfeed 
it means that person is HIV positive, so I am not ready to face such challenges of being neglected by people in the community" (participant 9-32 years).

Mgongo, Mosha and Uriyo's study reported insufficient support at a community level, which they felt was critical for sustaining the advice on infant feeding and this was in line with the findings by Chinkonde, Hem and Sundby $[18,19]$. When the women stopped breastfeeding at six months for example, it was assumed that they were doing so because they were HIV positive. Consequently, they were blamed for that and were treated like loose women even though it may have been their husbands who brought the HIV into the marriage [19].

\section{Mixed feeding reactions}

Findings in this study have reflected that HIV positive mothers have mixed reactions regarding feeding options for their children and this correlates with findings by Ngwende et al., [20]. The views of participants' regarding mixed feeding were:

"One part of me wanted to exclusively breastfeed my baby, but the other was reluctant and hence I ended up giving my baby both the breast and the formula added in the soft porridge. My baby tested positive for HIV I will never forgive myself for this" (participant 5-22 years).

"Getting the messages at the clinic regarding breastfeeding sounds simple and easy to apply but in real life is not possible; you can't look at your baby cry and not give additional food" (participant 7-30 years).

Exclusive breastfeeding by mothers is a challenge to adhere to during the first six months for most women and therefore predisposes the infant to the risk of HIV infection, especially for mothers who have responsibilities that take them away from their children during the day [20]. A study that investigated infant feeding choices and experiences of HIV positive mothers from two Ghanaian districts, found social pressure and local norms as factors that influenced mothers' decisions to mix feed their children [8]. One of the UNAIDS strategies of ending HIV infection by 2020 includes elimination of new HIV infections among children and their mother's health and well-being is sustained [21].

\section{Theme 2: Physical problems experienced by mothers who are breastfeeding}

Women reported the presence of physical problems that also prevent them from breastfeeding properly, such as problems in the breasts and ill health of the mother.

\section{Sore breasts make it difficult to breastfeed}

Participants revealed problems of the breast as being aggravating factors regarding lack of proper feeding in infants, resulting in mixed feeding and increased risk of HIV transmission from the mother to the baby.

"Most of the time my breasts are so sore and painful and I feel breastfeeding would kill me and as such I would prepare soft porridge and give it to my baby and later I give the baby water to wash off the soft porridge" (participant 11-20 years).

"Maybe you were fortunate you have never experienced painful breasts, the type of pain is horrible and when the baby sucks you feel as if a sharp pin is striking your heart and the worst part is that while breastfeeding the breast milk from the sore breast might contain blood which puts my baby at risk of getting HIV" (participant 16-30 years).
The findings from this study were comparable to findings from previous studies and have reflected that breastfeeding the baby from an infected breast increases the risk of HIV transmission from the mother to the baby. According to Coovadia and Bland, breastfeeding with sore nipples increases the risk of HIV transmission [16]. This was supported by Semrau, Kuhn, Brooks et al., that babies breastfeeding from breasts with infections increases the risk of HIV transmission from the mother to the baby [22].

\section{Severe illness resulting in lack of energy hampers feeding op-} tions for the baby

Some participants showed that HIV poses many challenges to them with regard to breastfeeding and ill health because when mothers are critically ill, breastfeeding the baby becomes very difficult without support.

"When I'm sick I struggle a lot failing to take of the baby and my in-laws would ask me what I am suffering from, I don't have any choice but lie at the same time who knows my baby can be infected by HIV in the process" (participant 11-20 years).

"I was very weak and dizzy I could not even think about breastfeeding the baby and my husband would just attach her to my breast only when she cries (participant 7-30 years).

Embree et al., identified maternal ill health as a factor considered in the analysis both as a clinical marker of maternal immunosuppression and as a potential source of infant fungal colonization, which increases the risk of mother to child transmission [23]. Maternal immunosuppression increases the risk of mother to child transmission during the postnatal period in babies who are being breastfed.

\section{Conclusion}

The findings have highlighted many serious challenges experienced by women who are HIV positive regarding breastfeeding, which predispose their babies to HIV infections. Culture and the role played by in-laws are key significant findings in this study. Some of the challenges are external, coming from their families and the community as well as from within themselves such as ill health attributed to HIV progression. It is imperative the health education on infant feeding practices should take cognizance of this. This will help in achieving the 90-90-90 strategy and the mandate to fast track the end to HIV by addressing babies and ensuring an HIV negative generation from women is infected by HIV.

\section{Acknowledgement}

The researcher would like to acknowledge the support received from National Research Foundation and Medical Research Council for funding the study as well the contributions made by the promoters.

\section{References}

1. WHO/UNAIDS (2004) HIV and infant feeding: guidelines for decision-makers Maternal, newborn, child and adolescent health, WHO, Geneva, Switzerland.

2. Avert (2017) Prevention of Mother-To-Child Transmission (PMTCT) of HIV. Global information and education on HIV and AIDS, AVERT, UK.

3. Coovadia H, Kindra G (2008) Breastfeeding to prevent HIV transmission in infants: balancing pros and cons. Curr Opin Infect Dis 21: 11-15.

4. Health Systems Trust (2010) A leading resource on health systems and primary health care in southern Africa. Maternal, child and infant feeding. HST publications, South Africa. 
5. Muluye D, Woldeyohannes D, Gizachew M, Tiruneh M (2012) Infant feeding practice and associated factors of HIV positive mothers attending prevention of mother to child transmission and antiretroviral therapy clinics in Gondar Town health institutions, Northwest Ethiopia. BMC Public Health 12: 240.

6. Creswell JN (2011) Educational Research: Planning, Conducting, and Evaluating Quantitative and Qualitative Research (4th Edn). Pearson, England, UK.

7. Seidel G, Sewpaul V, Dano B (2000) Experiences of breastfeeding and vulnerability among a group of HIV-positive women in Durban, South Africa. Health Policy Plan 15: 24-33.

8. Laar AK, Ampofo W, Tuakli JM, Quakyi IA (2009) Infant feeding choices and experiences of HIV-positive mothers from two Ghanaian districts. Journal of AIDS and HIV Research 1: 23-33.

9. Wringe A, Roura M, Urassa M, Busza J, Athanas V, et al. (2009) Doubts, denial and divine intervention: understanding delayed attendance and poor retention rates at a HIV treatment programme in rural Tanzania. AIDS Care 21: $632-637$.

10. Piper K, Enah C, Daniel M (2014) Black southern rural adolescents' HIV stigma, denial, and misconceptions and implications for HIV prevention. J Psychosoc Nurs Ment Health Serv 52: 50-56.

11. Lazarus R, Struthers H, Violari A (2013) Promoting safe infant feeding practices - the importance of structural, social and contextual factors in Southern Africa. J Int AIDS Soc 16: 18037.

12. Amir LH (2011) Social theory and infant feeding. Int Breastfeed J 6: 7.

13. Shapiro RL, Thior I, Gilbert PB, Lockman S, Wester C, et al. (2006) Maternal single-dose nevirapine versus placebo as part of an antiretroviral strategy to prevent mother-to-child HIV transmission in Botswana. AIDS 20: 1281-1288.

14. Omari AA, Luo C, Kankasa C, Bhat GJ, Bunn J (2003) Infant-feeding practices of mothers of known HIV status in Lusaka, Zambia. Health Policy Plan 18: $156-162$.
15. Leshabari SC, Blystad A, Moland KM (2007) Difficult choices: Infant feeding experiences of HIV-positive mothers in northern Tanzania. SAHARA $J$ 4: $544-555$

16. Coovadia HM, Rollins NC, Bland RM, Little K, Coutsoudis A, et al. (2007) Mother-to-child transmission of HIV-1 infection during exclusive breastfeeding in the first 6 months of life: an intervention cohort study. Lancet 369: 11071116.

17. Horvath T, Madi BC, luppa IM, Kennedy GE, Rutherford G, et al. (2009) Interventions for preventing late postnatal mother-to-child transmission of HIV. Cochrane Database Syst Rev.

18. Mgongo M, Mosha MV, Uriyo JG, Msuya SE, Stray-Pedersen B (2013) Prevalence and predictors of exclusive breastfeeding among women in Kilimanjaro region, Northern Tanzania: a population based cross-sectional study. Int Breastfeed J 8: 12

19. Chinkonde, JR, Hem MH, Sundby J (2012) HIV and infant feeding in Malawi: public health simplicity in complex social and cultural contexts. BMC Public Health 12: 700 .

20. Ngwende S, Gombe NT, Midzi S, Tshimanga G, Shambira G, et al. (2013) Factors associated with HIV infection among children born to mothers on the prevention of mother to child transmission programme at Chitungwiza Hospital, Zimbabwe. BMC Public Health 13: 1181.

21. UNAIDS (2016-2021) On the Fast-Track to end AIDS. 90-90-90 Strategy, UNAIDS, Geneva, Switzerland.

22. Semrau K, Kuhn L, Brooks DR, Cabral H, Sinkala M, et al. (2011) Exclusive breastfeeding, maternal HIV disease, and the risk of clinical breast pathology in HIV-infected, breastfeeding women. Am J Obstet Gynecol 205: 344-348.

23. Embree JE, Njenga S, Datta $P$, Nagelkerke NJ, Ndinya-Achola JO, et al. (2000) Risk factors for postnatal mother-child transmission of HIV-1. AIDS 14: $2535-2541$ 